\title{
Gamma Knife Radiosurgery Versus Transcatheter Arterial Chemoembolization for Hepatocellular Carcinoma With Portal Vein Tumor Thrombus: a Propensity Score Matching Study
}

$\mathrm{Ke} \mathrm{Su}$

The Affiliated Hospital of Southwest Medical University

Tao Gu

The Affiliated Hospital of Southwest Medical University

$\mathrm{KeXu}$

The Affiliated Hospital of Southwest Medical University

Jing Wang

The Affiliated Hospital of Southwest Medical University

Hongfei Liao

The Affiliated Hospital of Southwest Medical University

Xueting Li

The Affiliated Hospital of Southwest Medical University

Lianbin Wen

Sichuan Province People's Hospital: Sichuan Academy of Medical Sciences and Sichuan People's Hospital

\section{Yanqiong Song}

Sichuan Cancer Hospital and Institute

Jiaqi Zhong

The Affiliated Hospital of Southwest Medical University

\section{Bingsheng He}

The Affiliated Hospital of Southwest Medical University

Xin Liu

The Affiliated Hospital of Southwest Medical University

Jie He

The Affiliated Hospital of Southwest Medical University

Yanlin Liu

The Affiliated Hospital of Southwest Medical University

Qi Li

The Affiliated Hospital of Southwest Medical University

Xunjie Feng 
Southwest Medical University

\section{Siyu Chen}

Southwest Medical University

\section{Binbin Yang}

Southwest Medical University

\section{Weihong Huang}

Southwest Medical University

\section{Hongping Jin}

Southwest Medical University

\section{Xiaotong Luo}

Southwest Medical University

\section{Teng Hu}

The Affiliated Hospital of Southwest Medical University

\section{Jiali Chen}

The Affiliated Hospital of Southwest Medical University

\section{Zhenying Wu}

The Affiliated Hospital of Southwest Medical University

\section{Simin Lu}

The Affiliated Hospital of Southwest Medical University

\section{Jianwen Zhang}

The Affiliated Hospital of Southwest Medical University

\section{Mingyue Rao}

The Affiliated Hospital of Southwest Medical University

\section{Yunchuan Xie}

The Affiliated Hospital of Southwest Medical University

\section{Jing Wang}

The Affiliated Hospital of Southwest Medical University

\section{Xiaoning Zhu}

The Affiliated Hospital of Southwest Medical University

\section{Lan Chen}

The Affiliated Hospital of Southwest Medical University

\section{Bo Li}

The Affiliated Hospital of Southwest Medical University

\section{Song Su}

The Affiliated Hospital of Southwest Medical University

\section{Xiaoli Yang}

The Affiliated Hospital of Southwest Medical University

\section{Juan Wang}

The Affiliated Hospital of Southwest Medical University 


\section{Hao Zeng}

The Affiliated Hospital of Southwest Medical University

\section{Pan Wang}

The Affiliated Hospital of Southwest Medical University

\section{Min Yan}

The Affiliated Hospital of Southwest Medical University

\section{Xiaojing Chen}

The Affiliated Hospital of Southwest Medical University

\section{Kun $\mathrm{He}$}

The Affiliated Hospital of Southwest Medical University

Yunwei Han ( $\square$ Lanpaoxiansheng@126.com )

Shandong University School of Basic Medical Sciences https://orcid.org/0000-0002-0942-9095

\section{Research Article}

Keywords: Gamma knife radiosurgery, radiotherapy, transcatheter arterial chemoembolization, hepatocellular carcinoma, primary liver cancer, portal vein tumor thrombus, propensity score matching, overall survival, Cheng's classification, advanced stage

Posted Date: January 3rd, 2022

DOI: https://doi.org/10.21203/rs.3.rs-1193272/v1

License: (c) (1) This work is licensed under a Creative Commons Attribution 4.0 International License. Read Full License 


\section{Abstract}

\section{Background}

The optimal management for hepatocellular carcinoma (HCC) patients with portal vein tumor thrombus (PVTT) is unclear. This study aimed to investigate the efficacy of Gamma knife radiosurgery (GKR) versus transcatheter arterial chemoembolization (TACE) in HCC patients with PVTT.

\section{Methods}

This retrospective study included 572 HCC patients with PVTT (GKR, 207; TACE, 365). Propensity score matching (PSM) analysis identified 188 matched pairs of patients. The primary endpoint was overall survival (OS).

\section{Results}

Before PSM, the GKR group demonstrated significantly median OS (mOS) than TACE group (17.4 vs. 8.0 months, $p<0.001)$. In the subgroup analysis, GKR was associated with significantly longer OS for patients with PVTT II-IV (17.5 vs. 8.2 months, $p<0.001 ; 19.3$ vs. 7.4 months, $p<0.001 ; 14.5$ vs. 6.6 months, $p=0.008$, respectively) and comparable OS for patients with PVTT I. After PSM, the GKR group had also a longer OS (mOS) than TACE group (15.8 vs. 9.8 months, $p<0.001)$. In the subgroup analysis, the GKR group demonstrated superior mOS for patients with PVTT II-IV (all P $<0.05$ ) and comparable OS for patients with PVTT I.

\section{Conclusions}

GKR was associated better OS than TACE in HCC patients with PVTT, especially for patients with PVTT IIIV.

\section{Introduction}

Hepatocellular carcinoma (HCC) is characterized by high morbidity and death (1). HCC is prone to invade the portal vein system and can cause portal vein tumor thrombus (PVTT), which is a poor prognostic factor and results in a median overall survival (mOS) of only 2-4 months (2-4). According to current literature, liver resection (LR) is the preferred treatment in PVTT I/Il patients with Child-Pugh A and Eastern Cooperative Oncology Group performance status (ECOG PS) 0-1 $(5,6)$. However, for patients with other types of PVTT, there is still controversy regarding the best therapeutic approaches (7).

With the advancement of local therapeutic techniques, extremely transcatheter arterial chemoembolization (TACE), radiofrequency ablation (RFA), radiotherapy including gamma knife radiosurgery (GKR) and stereotactic body radiation therapy (SBRT) have become feasible. HCC patients with PVTT receiving TACE extended months mOS to $4-10$ months $(8,9)$. But as an invasive treatment, TACE has a great impact on the life of the patient and carries risks such as liver failure. GKR is a form of 
external radiotherapy, it can allow delivery of ablative doses of radiation with low toxicity. A retrospective study of small sample of patients reported that the mOS of HCC patients with PVTT who received GKR was 6.1 months ( $95 \% \mathrm{Cl}: 4.7-7.5)(10)$. In addition, a study by Xiaojie and colleagues reported that patients with HCC-derived PVTT who received GKR combined with TACE had longer OS than those treated with TACE alone (11).

Although HCC patients with PVTT who do not undergo surgery are candidates for both GKR and TACE, to the best of our knowledge, no data comparing these modalities is available. Therefore, we conducted this study to compare the effectiveness of GKR versus TACE for HCC patients with PVTT.

\section{Materials And Methods}

\section{Patients}

This is a multicentre, retrospective, cohort analysis of HCC patients with PVTT treated with GKR or TACE between June, 2015 and July, 2021.

The inclusion criteria were as follows: (1) patients aged 18 years or older; (2) histological or cytological diagnosis of HCC; (3) confirmation of PVTT by contrast-enhanced computed tomography (CT) or magnetic resonance imaging (MRI); (4) measurable lesions assessed as per Response Evaluation Criteria in Solid Tumors RECIST Version 1.1 (RECIST 1.1); (5) Child-Pugh class of A or B; (6) score of 0 to 1 according to ECOG PS; (7) tumor surgery deemed unfeasible, difficult, or unsuitable.

The exclusion criteria were as follows: (1) patients concomitantly received other treatments such as sorafenib, TACE plus GKR, hepatic resection, or radiofrequency ablation; (2) patients with obstinate ascites, hepatic encephalopathy; (3) patients with cancers other than HCC; (4) inadequate clinical data; (5) patients who had used any treatment of anti-tumor within 12 weeks prior to the first treatment in this study.

\section{Classification of PVTT}

We followed the Cheng's classification for PVTT and classified the types of PVTT according to four levels: (a) type I was defined as PVTT in the segmental or sectoral branches of the portal vein or above; (b) type II was defined as PVTT in the right/left portal vein; (c) type III was defined as PVTT in the main portal vein; and (d) type IV was defined as PVTT in the superior mesenteric vein. Type 10 represented tumor thrombus found only under microscopy observation (12-14).

\section{Treatment Protocol}

The decision to offer GKR, TACE for HCC was made by the Hospital HCC Expert Team including hepatologists, radiation oncologists, surgeons, interventional radiologists, medical oncologists and pathologists. All patients signed informed consents before they received any treatment. 
GKR was performed using Treatment Planning System (TPS) by two or three radiation oncologists who delineated the irradiation area as per contrast-enhanced CT scan. Delineation of the gross target volume (GTV) including the primary liver tumor and tumor thrombosis was done based on the image technology. The radiation oncologists defined a $5-10 \mathrm{~mm}$ margin around the GTV as the planning target volume (PTV) by TPS. The median tumor margin dose was $42 \mathrm{~Gy}$ (range, 39 to $42 \mathrm{~Gy}$ ), with a median isodose line of $50 \%$ (range, $50 \%$ to $60 \%$ ). Dose-volume histograms were used to protect adjacent normal tissues and the liver, as delineated during the target planning process. The course of GKR treatment was divided into 2 cycles with 7 times per course (once a day). The radiation oncologists revised the treatment plan by CT after the first course of treatment, and ahead of the second course of treatment.

\section{TACE}

TACE was performed by delivering an emulsion of lipiodol (10-20 ml) and one or more chemotherapeutic agents, such as cisplatin, or cisplatin and mitomycin-C, or Fluorouracil. The delivery was performed into the selected vessels after a microcatheter was advanced into the hepatic arterial circulation to the most distal tumor-feeding vessel, followed by embolization using gelatin sponge particles or other embolic materials if deemed necessary.

Repeated TACE procedures were performed in the case of multiple lesions or large lesions. The decision to perform multiple TACE was made by HCC Expert Team.

\section{Patient Follow-Up and Data Collection}

Post-treatment follow-ups were conducted every 2-3 months and entailed comparison of CT, or MRI and laboratory tests at baseline. Laboratory tests included biochemical and hematologic analyses, such as pro-thrombin time; complete blood cell count; and measurements of a-fetoprotein (AFP), total bilirubin, alkaline phosphatase (ALP), alanine aminotransferase (ALT), and aspartate aminotransferase (AST) levels. Patients with recurrent tumor on CT or MRI images underwent second-line treatment.

\section{Statistical Analysis}

Categorical variables were analyzed using $\chi 2$ and McNemar analysis. One-to-one propensity score matching (PSM) was adopted to reduce selection bias and confounding factors resulting from different co-variable distribution among GKR and TACE groups. Independent variables included in the propensity model were sex, age, Child-Pugh classification, tumor number, tumor size, AFP levels, ALP levels, platelet levels, ALT levels, leukocyte levels, type of PVTT, HBV infection, HCV infection, drinking history, lymph node metastasis, extrahepatic metastasis, and previous therapy status. Survival was computed as the interval between the date of GKR or TACE and the date of death for any reason, with censoring at the date of last follow-up in surviving patients. Kaplan-Meier method with log-rank test was utilized to compare long-term survival distribution. In exploratory subgroup analyses, the OS of GKR and TACE in predefined subgroups was assessed using Cox proportional hazard models presented in a forest plot; forest plots 
show factors associated with OS. Univariate and multivariable Cox analyses of all data were done to confirm significant predictors for OS. Factors with $\mathrm{P}$ values less than 0.05 in univariate analysis were introduced into the multivariate Cox proportional hazards model to determine the adjusted hazard ratios (HRs) and 95\% confidence intervals (Cls). All statistical analyses were conducted with SPSS for Windows (version 26.0). Two tailed $P$ value $₫ 0.05$ were considered as statistically significant.

\section{Results}

\section{Patient characteristics of the pooled and matched cohorts}

Between June, 2015 and July, 2021, 4563 patients were diagnosed with HCC. Among them, 3991 patients did not meet the eligibility criteria and were excluded. We included in this study 207 patients who received GKR and 365 patients who received TACE (Supplementary Fig. 1). Before matching, the median follow-up period was 25.5 months $(95 \% \mathrm{Cl} 19.1-31.9)$ for the GKR group and 20.8 months (95\% $\mathrm{Cl} 17.8-23.8)$ for the TACE group. Patients who underwent TACE had more multiple tumors $(P=0.012)$, larger tumors $(P=$ 0.007), higher AFP levels $(P=0.001)$, more lymph node metastases $(P=0.010)$, worse PVTT type $(P=$ 0.016) than did those patients who underwent GKR. Patients in the GKR group had experienced a higher number of previous therapies $(P<0.001)$ than patients in the TACE group (Table 1$)$. 
Table 1

Baseline characteristics of the patients before and after PSM

\begin{tabular}{|c|c|c|c|c|c|c|}
\hline & Before PSN & & & After PSM & & \\
\hline Variable & GKR & TACE & $\mathrm{P}$ & GKR & TACE & $P$ \\
\hline patients & 207 & 365 & & 188 & 188 & \\
\hline Male sex & 183(88.4) & $320(87.7)$ & 0.795 & $164(87.2)$ & $170(90.4)$ & 0.405 \\
\hline Age $\geq 60$ years & 77(37.2) & $117(32.1)$ & 0.212 & $65(34.6)$ & $70(37.2)$ & 0.630 \\
\hline Child-Pugh B & $59(28.5)$ & $116(31.8)$ & 0.414 & $56(29.8)$ & $60(31.9)$ & 0.724 \\
\hline Number of tumors $\geq 2$ & $155(74.9)$ & $305(83.6)$ & 0.012 & $147(78.2)$ & $157(83.5)$ & 0.220 \\
\hline Tumor size $\geq 5 \mathrm{~cm}$ & $151(72.9)$ & $301(82.5)$ & 0.007 & $142(75.5)$ & $155(82.4)$ & 0.085 \\
\hline Serum AFP $\geq 400 \mathrm{ng} / \mathrm{ml}$ & $97(46.9)$ & $223(61.1)$ & 0.001 & $95(50.5)$ & $108(57.4)$ & 0.154 \\
\hline ALP levels $\geq 125 \mathrm{U} / \mathrm{L}$ & $135(65.2)$ & $270(74.0)$ & 0.027 & $127(67.6)$ & 133(70.7) & 0.556 \\
\hline Platelet count $\geq 100 \times 109 / L$ & 153(73.9) & $267(73.2)$ & 0.843 & 139(73.9) & 133(70.7) & 0.550 \\
\hline ALT levels $\geq 40 \mathrm{U} / \mathrm{L}$ & $122(58.9)$ & $227(62.2)$ & 0.443 & $112(59.6)$ & $112(59.6)$ & 1.000 \\
\hline leukocyte $\geq 4 \times 10^{9} / \mathrm{L}$ & 163(78.7) & $292(80.0)$ & 0.720 & $151(80.3)$ & $151(80.3)$ & 1.000 \\
\hline Number of TACE $\geq 2$ & - & $80(21.9)$ & & - & $40(21.3)$ & \\
\hline Cheng's type of PVTT & & & 0.016 & & & 0.139 \\
\hline I & $44(21.3)$ & $45(12.3)$ & & $35(18.6)$ & $29(15.4)$ & \\
\hline II & 63(30.4) & $147(40.3)$ & & $58(30.9)$ & $78(41.5)$ & \\
\hline III & $67(32.4)$ & 118(32.3) & & $62(33.0)$ & $55(29.3)$ & \\
\hline IV & $33(15.9)$ & $55(15.1)$ & & $33(17.6)$ & $26(13.8)$ & \\
\hline \multicolumn{7}{|l|}{ Etiology } \\
\hline HBV & $138(66.7)$ & $221(60.5)$ & 0.146 & $123(65.4)$ & $118(62.8)$ & 0.649 \\
\hline $\mathrm{HCV}$ & $4(1.9)$ & $9(2.5)$ & 0.681 & $4(2.1)$ & $4(2.1)$ & 1.000 \\
\hline Alcohol & $89(43)$ & $147(40.3)$ & 0.525 & $81(43.1)$ & $76(40.4)$ & 0.691 \\
\hline Lymph node metastasis & $103(49.8)$ & $222(60.8)$ & 0.010 & $93(49.5)$ & $104(55.3)$ & 0.289 \\
\hline Extrahepatic metastases & $58(28.0)$ & 102(27.9) & 0.985 & $48(25.5)$ & $57(30.3)$ & 0.368 \\
\hline Lung & $29(14.0)$ & 71(19.5) & & 25(13.3) & $40(21.3)$ & \\
\hline Bone & $24(11.6)$ & $17(4.7)$ & & 16(8.5) & $8(4.3)$ & \\
\hline
\end{tabular}




\begin{tabular}{|c|c|c|c|c|c|c|}
\hline \multirow[b]{2}{*}{ other } & \multicolumn{3}{|c|}{ Before PSM } & \multicolumn{3}{|c|}{ After PSM } \\
\hline & $19(9.2)$ & $28(7.7)$ & & $17(9.0)$ & $17(9.0)$ & \\
\hline Previous therapy & $102(49.3)$ & $74(20.3)$ & $<0.001$ & $85(45.2)$ & $73(38.8)$ & 0.210 \\
\hline Systemic therapy & $45(21.7)$ & $9(2.5)$ & & $40(21.3)$ & $9(4.8)$ & \\
\hline Liver resection & $44(21.3)$ & $24(6.6)$ & & $34(18.1)$ & $23(12.2)$ & \\
\hline radiotherapy & 18(8.7) & 0 & & $14(7.4)$ & 0 & \\
\hline TACE & $51(24.6)$ & $51(14.0)$ & & $39(20.7)$ & $51(27.1)$ & \\
\hline RFA & $14(6.8)$ & $4(1.1)$ & & $11(5.9)$ & $4(2.1)$ & \\
\hline \multicolumn{7}{|c|}{$\begin{array}{l}\text { Abbreviations: PSM, propensity score matching; AFP, alpha fetoprotein; ALP, alkaline phosphatase; } \\
\text { ALT, alanine aminotransferase; PVTT, portal vein tumor thrombus; HBV, hepatitis B virus; HCV, } \\
\text { hepatitis C virus; GKR, gamma knife radiosurgery; TACE, transcatheter arterial chemoembolization; } \\
\text { RFA, radiofrequency ablation. }\end{array}$} \\
\hline
\end{tabular}

After performing PSM, we identified 188 matched pairs of patients with comparable patient and tumor characteristics. In this matched cohort, patients in the GKR and TACE groups were not significantly different with regards to any patient or tumor covariates at baseline (Table 1). The median follow-up time was 25.9 months $(95 \% \mathrm{Cl} 20.2-31.6)$ in the GKR group and 20.8 months $(95 \% \mathrm{Cl} 13.7-27.9)$ in the TACE group.

\section{Overall Survival Analyses in the pooled and matched Cohorts}

At the cutoff date (September 1, 2021), In the pooled cohort, 105 patients $(50.7 \%)$ in the GKR group and $253(69.3 \%)$ in the TACE group had died. The mOS was 17.4 months (14.2-20.6) in the GKR group and 8.0 months (6.4-9.6) in the TACE group ( $p<0.001$, Fig. 1A). The cumulative OS rates at 12, 18, and 24 months were $62.9 \%, 46.1 \%$, and $40.4 \%$ in the GKR group, and $39.3 \%, 30.7 \%$, and $25.2 \%$ in the TACE group, respectively. The OS rates were significantly higher in patients in the GKR group than in those in the TACE group ( $p<0.001)$. In subgroup analysis according to the type of PVTT, patients in the GKR group had a significantly longer OS than those patients with PVTT type II, III and IV in the TACE group (17.5 vs. 8.2 months, $p<0.001$, Fig. 2C; 19.3 vs. 7.4 months, $p<0.001$, Fig. $2 E ; 14.5$ vs. 6.6 months, $p=0.008$, Fig. 2G; respectively). However, there was no significant difference in the OS between the two groups of patients with PVTT type I (14.4 vs. 12.5 months, $p=0.396$, Fig. $2 A$ ).

In the matched cohort, 102 (54.3\%) patients in the GKR group patients and $126(67.0 \%)$ in the TACE group patients died. The GKR group had a mOS of 15.8 (13.4-18.2) months, which was higher than that of 9.8 (6.1-13.5) months in the TACE group ( $p<0.001$, Fig. 1B). The cumulative OS rates at 12, 18, and 24 months were $59.7 \%, 41.9 \%$, and 36.8 in the GKR group, and $45.1 \%, 34.8 \%$, and $27.4 \%$ in the TACE group ( $p$ $<0.001$ ), respectively. Patients with PVTT type II, III and IV in the GKR group had also a significantly 
longer OS than their counterparts the TACE group (17.5 vs. 9.8 months, $p=0.003$, Fig. 2D; 17.2 vs. 7.8 months, $p=0.028$, Fig. $2 F ; 14.5$ vs. 5.5 months, $p=0.043$, Fig. $2 \mathrm{H}$; respectively). There was no significant difference in the OS between the two groups of patients with PVTT type I (11.2 vs. 14.4 months, $p=$ 0.703 , Fig. 2B).

\section{Exploratory subgroup analysis of associated factors in the matched Cohorts}

In exploratory subgroup analyses of OS after PSM (Supplementary Fig. 2), GKR seemed most beneficial in male patients $(p=0.002)$; those younger than 60 years $(p=0.001)$; tumor size $\geq 5 \mathrm{~cm}(p=0.002) ; \operatorname{ALP}$ $\geq 125 \mathrm{U} / \mathrm{L}(\mathrm{P}<0.001)$; ALT $\geq 40 \mathrm{U} / \mathrm{L}(\mathrm{P}<0.001)$; leukocyte $\geq 4000 / \mu \mathrm{L}(\mathrm{p}=0.002)$; PVTT type II-IV $(\mathrm{p}=$ $0.003, p=0.030, p=0.048$, respectively); non-HCV $(p=0.001)$; non-lymh node metastases $(p<0.001)$; and non-extrahepatic metastases $(p<0.001)$.

\section{Factors Associated with Overall Survival in the matched Cohorts}

We performed univariate and multivariate Cox regression analyses to confim prognostic independent factors after PSM. The child A, GKR, number of tumors $<2$, tumor diameter $<5 \mathrm{~cm}$, and ALP $<125 \mathrm{U} / \mathrm{L}$ were all confirmed as independent positive prognostic factor for OS (all $p<0.005$, Table 2 ). 
Table 2

univariate and multivariate Cox regression analysis of overall survivalafter PSM

Univariable Cox regression

\begin{tabular}{|c|c|c|c|c|c|c|}
\hline Variable & $\mathrm{HR}$ & $95 \% \mathrm{Cl}$ & $P$ & $\mathrm{HR}$ & $95 \% \mathrm{Cl}$ & $P$ \\
\hline Sex (male/female) & 1.004 & $\begin{array}{l}0.667- \\
1.511\end{array}$ & 0.984 & & & \\
\hline Age $(\geq 60 /<60$ years $)$ & 0.813 & $\begin{array}{l}0.617- \\
1.072\end{array}$ & 0.143 & & & \\
\hline Child-Pugh class (B/A) & 1.657 & $\begin{array}{l}1.256- \\
2.184\end{array}$ & $<0.001$ & 1.426 & $\begin{array}{l}1.074- \\
1.894\end{array}$ & 0.014 \\
\hline Number of tumor $(\geq 2 /<2)$ & 1.780 & $\begin{array}{l}1.235- \\
2.566\end{array}$ & 0.002 & 1.497 & $\begin{array}{l}1.035- \\
2.164\end{array}$ & 0.032 \\
\hline Tumor diameter $(\geq 5 /<5 \mathrm{~cm})$ & 2.282 & $\begin{array}{l}1.563- \\
3.329\end{array}$ & $<0.001$ & 1.896 & $\begin{array}{l}1.292- \\
2.781\end{array}$ & 0.001 \\
\hline $\operatorname{AFP}(\geq 400 /<400 \mathrm{ng} / \mathrm{ml})$ & 1.266 & $\begin{array}{l}0.973- \\
1.646\end{array}$ & 0.079 & & & \\
\hline $\operatorname{ALP}(\geq 125 /<125 \mathrm{U} / \mathrm{L})$ & 1.95 & $\begin{array}{l}1.439- \\
2.644\end{array}$ & $<0.001$ & 1.642 & $\begin{array}{l}1.194- \\
2.258\end{array}$ & 0.002 \\
\hline Platelet $(<100000 / \geq 100000 / \mu \mathrm{L})$ & 1.267 & $\begin{array}{l}0.938- \\
1.713\end{array}$ & 0.123 & & & \\
\hline $\operatorname{ALT}(\geq 40 /<40 U / L)$ & 1.223 & $\begin{array}{l}0.936- \\
1.597\end{array}$ & 0.14 & & & \\
\hline leukocyte $(<4000 / \geq 4000 / \mu \mathrm{L})$ & 1.14 & $0.818-1.59$ & 0.439 & & & \\
\hline Cheng's type of PVTT & & & 0.641 & & & \\
\hline I & 1.000 & & & & & \\
\hline ॥ & 1.045 & $\begin{array}{l}0.710- \\
1.538\end{array}$ & 0.825 & & & \\
\hline III & 1.149 & $\begin{array}{l}0.776- \\
1.703\end{array}$ & 0.488 & & & \\
\hline IV & 1.294 & $\begin{array}{l}0.824- \\
2.030\end{array}$ & 0.263 & & & \\
\hline HBV (positive/negative) & 1.199 & $\begin{array}{l}0.911- \\
1.578\end{array}$ & 0.195 & & & \\
\hline HCV (positive/negative) & 1.166 & $\begin{array}{l}0.549- \\
2.476\end{array}$ & 0.689 & & & \\
\hline Alcoholism (positive/negative) & 0.963 & $\begin{array}{l}0.740- \\
1.254\end{array}$ & 0.782 & & & \\
\hline
\end{tabular}




\begin{tabular}{|c|c|c|c|c|c|c|}
\hline \multirow[b]{2}{*}{ Lymph node metastasis (yes/no) } & \multicolumn{3}{|c|}{ Univariable Cox regression } & \multicolumn{3}{|c|}{ Multivariable Cox regression } \\
\hline & 1.21 & $\begin{array}{l}0.931- \\
1.572\end{array}$ & 0.153 & & & \\
\hline $\begin{array}{l}\text { Extrahepatic metastases } \\
\text { (yes/no) }\end{array}$ & 1.264 & $\begin{array}{l}0.954- \\
1.675\end{array}$ & 0.103 & & & \\
\hline Previous therapy (yes/no) & 0.939 & $\begin{array}{l}0.722- \\
1.222\end{array}$ & 0.641 & & & \\
\hline Treatment (GKR/TACE) & 0.628 & $\begin{array}{l}0.484- \\
0.816\end{array}$ & $<0.001$ & 0.664 & $\begin{array}{l}0.510- \\
0.864\end{array}$ & 0.002 \\
\hline \multicolumn{7}{|c|}{$\begin{array}{l}\text { Abbreviations: PSM, propensity score matching; HR, hazard ratio; PVTT, portal vein tumor thrombus; } \\
\text { AFP, alpha fetoprotein; ALP, alkaline phosphatase; ALT, alanine transaminase; HBV, hepatitis B virus; } \\
\text { HCV, hepatitis C virus; GKR, gamma knife radiosurgery; TACE, transcatheter arterial } \\
\text { chemoembolization. }\end{array}$} \\
\hline
\end{tabular}

\section{Discussion}

To the best of our knowledge, this is the first study to investigate the efficacy of GKR vs. TACE monotherapy in the treatment of HCC-PVTT patients. Treatment options are limited for HCC patients with PVTT, according to the NCCN guidelines (15). Previous studies reported that LR is the preferred treatment in PVTT I/II patients with good liver function but first-line treatment remains controversial in PVTT III/IV (5-7).

In this study, the TACE group had heavier tumor burden and higher AFP levels than the GKR group before PSM. Therefore,we performed PSM to reduce bias due to confounding in baseline characteristics between the two groups. We found that GKR was superior to TACE with respect to the survival time for patients with PVTT II, III, and IV (all P < 0.05). However, no significant difference in the OS before and after PSM was observed for patients with PVTT I. These results have implications for the treatment of HCC patients with PVTT.

Although both GKR and TACE are local therapies to induce tumor cell death, the two modalities differ substantially in their principles and processes of treatment, which might be underlie differences in treatment efficacy in HCC. A growing number of studies reported that TACE can be be safely conducted in patients with incomplete obstruction of the main portal vein or formation of abundant compensatory collateral branches of the portal vein, or recanalized portal vein by portal vein stenting despite complete obstruction (16-19). However, in cases of complete obstruction of the main portal vein by tumor thrombi with few collateral branches formed, TACE is contraindicated (16). Patients with PVTT type II, III, and IV often experience severe obstruction of the portal vein, which might be associated with the poor survival time of these patients in the TACE. In contrast, GKR, an external RT method, is the most widely used form of stereotactic radiosurgery in the USA due to its precision and the efficiency of radiation delivered in a single session $(20,21)$. With development of RT technology, radiation dosage of targeted regions can be increased while achieving protection of the adjacent healthy tissues, thereby making it suitable for HCC 
patients with all types of $\operatorname{PVTT}(7,22,23)$. In addition to sustaining local control with low toxicity, local RT also induce immunogenic cell death, increase the release of tumor antigens and activate the immune system $(10,24-26)$.

It is still unclear what the best therapeutic options for HCC patients with PVTT are. A retrospective study reported that HCC patients with PVTT who received GKR plus TACE had longer OS than those who received TACE alone (9.7 vs. 6.2 months, respectively; $p<0.001)(11)$. A study by Li and colleagues reported that mOS values of 8 vs. 10 months in the intensity-modulated radiation therapy (IMRT) and SBRT groups $(p=0.165)(27)$. In addition, Silva et al. conducted a meta-analysis and reported that the mOS of HCC patients who received TACE was 8 months (19). In our study, patients in the GKR group experienced longer survival time than those in the TACE after PSM (15.8 vs. 9.8 months, $p<0.001)$. At the same time, it is important to note that the total cost of GKR and TACE alone are similar in China. And due to non-invasion of GKR, patients are more willing to accept the GKR rather than TACE. In summary, taking into consideration overall efficacy and costs, GKR appears to be a more feasible therapeutic option for HCC patients with PVTT.

Next, we explored the prognostic factors for HCC patients with PVTT and found that GKR was independently associated with long-term OS. In addition, the presence of multiple tumors, tumor diameter $\geq 5 \mathrm{~cm}, \mathrm{ALP} \geq 125 \mathrm{U} / \mathrm{L}$ and Child $\mathrm{B}$ were associated with inferior OS after PSM. These findings are in line with previous reports linking these factors with poor prognosis in HCC patients (28-30).

This study has several limitations. Firstly, this was a retrospective study and as such it is prone to potential bias. We conducted PSM to avoid selection bias, but we cannot exclude potential confounding factors. Secondly, the information on the adverse effects was limited. Finally, the sample size of the GKR group was relatively small, although our study had the largest HCC patients with PVTT ever reported to our knowledge.

\section{Conclusions}

In conclusion, in this multicenter retrospective study, GKR showed better OS than TACE in HCC patients with PVTT, especially in those with PVTT type II, III and IV. These findings have significant implications for the treatment of HCC patients with PVTT. Prospective randomized trials are needed to demonstrate potential benefit and safety of GKR.

\section{Declarations}

\section{Funding support}

None.

\section{Competing Interests}


The authors declare no conflict of interest regarding the content of this paper.

\section{Author Contributions}

Ke Su, Tao Gu, Ke Xu, Jing Wang, Hongfei Liao, Xueting Li, Lianbin Wen, Yanqiong Song, Jiaqi Zhong, Bingsheng He, Xin Liu, Jie He, Yanlin Liu, Qi Li, Xunjie Feng, Siyu Chen, Binbin Yang, Weihong Huang, Hongping Jin, Xiaotong Luo, Teng Hu, Jiali Chen, Zhenying Wu, Simin Lu, Jianwen Zhang, Mingyue Rao, Yunchuan Xie, Jing Wang, Xiaoning Zhu, Lan Chen, Bo Li, Song Su, Xiaoli Yang, Juan Wang, Hao Zeng, Pan Wang, Xiaojing Chen, and Min Yan collected the data. Yunwei Han and Kun He designed the research study. Ke Su, Tao Gu, Ke Xu, Kun He and Yunwei Han wrote the manuscript and analyzed the data. All authors approved the final version of the manuscript.

\section{Data Availability}

All data generated or analyzed during this study are included in this article and its supplementary material files. Further enquiries can be directed to the corresponding author (Lanpaoxiansheng @126.com).

\section{Animal Research (Ethics)}

This research did not involve animal experiments.

\section{Consent to Participate (Ethics)}

This retrospective study was approved by the Ethics Committee of The Affiliated Hospital of Southwest Medical University (approval number KY2020254) and complied with the standards of the Declaration of Helsinki. Written informed consent was waived because of the retrospective study.

\section{Consent to Publish (Ethics)}

The all authors confirmed that the work described has not been published before and it is not under consideration for publication elsewhere. Its publication has been approved by all co-authors and the Affiliated Hospital of Southwest Medical University.

\section{Plant Reproducibility}

None.

\section{Clinical Trials Registration}

The study was registered in the Chinese Clinical Trials Registry under the registration number ChiCTR2100051057

\section{References}


1. Sung H, Ferlay J, Siegel RL, Laversanne M, Soerjomataram I, Jemal A, et al. Global Cancer Statistics 2020: GLOBOCAN Estimates of Incidence and Mortality Worldwide for 36 Cancers in 185 Countries. CA Cancer J Clin. 2021;71(3):209-49.

2. Sun J, Shi J, Li N, Guo W, Wu M, Lau W, et al. Portal vein tumor thrombus is a bottleneck in the treatment of hepatocellular carcinoma. 2016;13(4):452-8.

3. Schöniger-Hekele M, Müller C, Kutilek M, Oesterreicher C, Ferenci P, Gangl AJG. Hepatocellular carcinoma in Central Europe: prognostic features and survival. 2001;48(1):103-9.

4. Bruix J, Sherman M, JH. Management of hepatocellular carcinoma: an update. 2011;53(3):1020-2.

5. Peng Z, Guo R, Zhang Y, Lin X, Chen M, Lau WJC. Hepatic resection versus transcatheter arterial chemoembolization for the treatment of hepatocellular carcinoma with portal vein tumor thrombus. 2012;118(19):4725-36.

6. Kokudo T, Hasegawa K, Matsuyama Y, Takayama T, Izumi N, Kadoya M, et al. Survival benefit of liver resection for hepatocellular carcinoma associated with portal vein invasion. 2016;65(5):938-43.

7. Cheng S, Chen M, Cai J, Sun J, Guo R, Bi X, et al. Chinese Expert Consensus on Multidisciplinary Diagnosis and Treatment of Hepatocellular Carcinoma with Portal Vein Tumor Thrombus (2018 Edition). 2020;9(1):28-40.

8. Zhu K, Chen J, Lai L, Meng X, Zhou B, Huang W, et al. Hepatocellular carcinoma with portal vein tumor thrombus: treatment with transarterial chemoembolization combined with sorafenib-a retrospective controlled study. 2014;272(1):284-93.

9. Kim J, Shim J, Yoon H, Ko H, Kim J, Gwon DJLiojotlAftSotL. Chemoembolization related to good survival for selected patients with hepatocellular carcinoma invading segmental portal vein. 2018;38(9):1646-54.

10. Lu X, Dong J, Ji L, Xiao L, Ling C, Zhou JJO. Tolerability and efficacy of gamma knife radiosurgery on hepatocellular carcinoma with portal vein tumor thrombosis. 2016;7(3):3614-22.

11. Lu X, Dong J, Ji L, Luo J, Cao H, Xiao L, et al. Safety and efficacy of TACE and gamma knife on hepatocellular carcinoma with portal vein invasion. 2016;65(4):715-6.

12. Shi J, Lai E, Li N, Guo W, Xue J, Lau W, et al. A new classification for hepatocellular carcinoma with portal vein tumor thrombus. 2011;18(1):74-80.

13. Xiang X, Lau W, Wu Z, Zhao C, Ma Y, Xiang B, et al. Transarterial chemoembolization versus best supportive care for patients with hepatocellular carcinoma with portal vein tumor thrombus: a multicenter study. 2019;45(8):1460-7.

14. Shi J, Lai E, Li N, Guo W, Xue J, Lau W, et al. Surgical treatment of hepatocellular carcinoma with portal vein tumor thrombus. 2010;17(8):2073-80.

15. Benson A, D'Angelica M, Abbott D, Abrams T, Alberts S, Anaya D, et al. Guidelines Insights: Hepatobiliary Cancers, Version 2.2019. 2019;17(4):302-10.

16. Zhou J, Sun H, Wang Z, Cong W, Wang J, Zeng M, et al. Guidelines for the Diagnosis and Treatment of Hepatocellular Carcinoma (2019 Edition). 2020;9(6):682-720. 
17. Leng J, Xu Y, Dong JJAjos. Efficacy of transarterial chemoembolization for hepatocellular carcinoma with portal vein thrombosis: a meta-analysis. 2016;86(10):816-20.

18. Xue T, Xie X, Zhang L, Yin X, Zhang B, Ren ZJBg. Transarterial chemoembolization for hepatocellular carcinoma with portal vein tumor thrombus: a meta-analysis. 2013;13:60.

19. Silva J, Berger N, Tsai S, Christians K, Clarke C, Mogal H, et al. Transarterial chemoembolization in hepatocellular carcinoma with portal vein tumor thrombosis: a systematic review and meta-analysis. 2017;19(8):659-66.

20. Parker T, Rigney G, Kallos J, Stefko S, Kano H, Niranjan A, et al. Gamma knife radiosurgery for uveal melanomas and metastases: a systematic review and meta-analysis. 2020;21(11):1526-36.

21. Park H, Wang E, Rutter C, Corso C, Chiang V, Yu JJJJon. Changing practice patterns of Gamma Knife versus linear accelerator-based stereotactic radiosurgery for brain metastases in the US. 2016;124(4):1018-24.

22. Hsieh C, Liu C, Shueng P, Chong N, Chen C, Chen M, et al. Comparison of coplanar and noncoplanar intensity-modulated radiation therapy and helical tomotherapy for hepatocellular carcinoma. 2010;5:40.

23. Tang Q, Li A, Yang G, Lai E, Zhou W, Jiang Z, et al. Surgical resection versus conformal radiotherapy combined with TACE for resectable hepatocellular carcinoma with portal vein tumor thrombus: a comparative study. 2013;37(6):1362-70.

24. Ohri N, Dawson L, Krishnan S, Seong J, Cheng J, Sarin S, et al. Radiotherapy for Hepatocellular Carcinoma: New Indications and Directions for Future Study. 2016;108(9).

25. Menon H, Ramapriyan R, Cushman T, Verma V, Kim H, Schoenhals J, et al. Role of Radiation Therapy in Modulation of the Tumor Stroma and Microenvironment. 2019;10:193.

26. Bujold A, Massey C, Kim J, Brierley J, Cho C, Wong R, et al. Sequential phase I and II trials of stereotactic body radiotherapy for locally advanced hepatocellular carcinoma. 2013;31(13):1631-9.

27. Li L, Zhou Y, Huang Y, Liang P, Liang S, Su TJHi. Stereotactic body radiotherapy versus intensitymodulated radiotherapy for hepatocellular carcinoma with portal vein tumor thrombosis. 2021;15(3):630-41.

28. Zhang X, Gao Y, Chen Z, Chen M, Li L, Wen T, et al. An Eastern Hepatobiliary Surgery Hospital/Portal Vein Tumor Thrombus Scoring System as an Aid to Decision Making on Hepatectomy for Hepatocellular Carcinoma Patients With Portal Vein Tumor Thrombus: A Multicenter Study. 2019;69(5):2076-90.

29. Yu M, Chan K, Lee C, Lee Y, Eldeen F, Chou H, et al. Alkaline phosphatase: does it have a role in predicting hepatocellular carcinoma recurrence? 2011;15(8):1440-9.

30. Wong T, Lee V, Law A, Pang H, Lam K, Lau V, et al. Prospective study of stereotactic body radiation therapy for hepatocellular carcinoma on waitlist for liver transplant. 2021.

\section{Figures}




\section{Figure 1}

(A) GKR had significantly improved overall survival compared to TACE before propensity score matching (17.4 vs. 8.0 months). (B) GKR had significantly improved overall survival compared to TACE in the matched cohort (15.8 vs. 9.8 months). Abbreviations: GKR, gamma knife radiosurgery; TACE, transcatheter arterial chemoembolization.

\section{Figure 2}

Overall survival curves for the patients with portal vein tumor thrombus (PVTT) type I (A), II (C), III (E), and IV (G) who received GKR and TACE are shown before propensity score matching. Overall survival curves for the patients with portal vein tumor thrombus (PVTT) type I (B), II (D), III (F), and IV (H) who received GKR and TACE are shown after propensity score matching. Abbreviations: GKR, gamma knife radiosurgery; TACE, transcatheter arterial chemoembolization.

\section{Supplementary Files}

This is a list of supplementary files associated with this preprint. Click to download.

- CopyrightTransferStatement28CTS29.docx

- Supplementary.docx 